\title{
Fruits: a key challenge in arid zones for generating added value, preserving biodiversity and addressing nutritional needs
}

With the emerging issue of climate change, arid zones are more than ever becoming a major challenge. In order to preserve some sustainable agricultural activity apart from extensive husbandry, there is an urgent need to look for drought-resistant species, to put emphasis on "deficit irrigation" and to promote crops that can give maximum added value to the farmers while at the same time enabling food security. Fruits and nuts are playing a major role in these areas: date palm, citrus, pomegranate, cactus, olive, apple, almonds, chestnuts, etc.

The last issue of Caravan, the review of the ICARDA (International Center for Agricultural Research in Dry Areas) on agriculture in dry areas strongly reflects this trend. Cactus is presented as a crop with a great range of opportunities for value addition: fruits, juice, pigments.

In Morocco, fruit trees are considered of considerable potential in remote mountain areas, gradually replacing potatoes, helping to connect the communities to profitable markets and leading to a significant reduction in poverty levels. In West Asia, rural communities are demonstrating that it pays to conserve vulnerable fruit species and, in other words, that value addition can help to conserve biodiversity. However, that does not mean closing the door to commercial fruit crops, such as olive plantations, that are expanding into marginal areas. A participatory approach is helping to reduce pressure on fragile resources

Such case studies from the NENA (Near East-North Africa) region are experiences that can be shared with other areas in the world, particularly Sub-Saharan and Southern Africa, where tropical and subtropical fruits trees can play a major role in terms of natural resource and biodiversity management, poverty alleviation, food security, and health of the local populations.

Dr. Jacky Ganry Chairman of the Section on Tropical and Subtropical Fruits ISHS 


\section{Les fruits : un enjeu majeur des zones arides pour générer des revenus, préserver la biodiversité et contribuer aux besoins nutritionnels}

Face à l'émergence des effets du changement climatique, les zones arides représentent plus que jamais un défi majeur. Afin de préserver durablement un minimum d'activité agricole en dehors d'un élevage extensif, il y a urgence à rechercher des espèces résistantes à la sécheresse, à gérer l'eau en situation de déficit tout en privilégiant des productions agricoles à forte valeur ajoutée pour les producteurs et en permettant d'assurer les besoins alimentaires. Les fruits charnus et à coque jouent un rôle important dans ces régions : palmier dattier, agrumes, grenadier, cactus, olivier, pommier, amandier, noyer, etc.

Le dernier numéro de Caravan, la revue de l'ICARDA (International Center for Agricultural Research in Dry Areas) sur l'agriculture en zones arides, reflète tout particulièrement cette tendance. Le cactus est présenté comme une culture présentant de multiples opportunités de valeur ajoutée : fruits, jus, colorants.

Au Maroc, les arbres fruitiers sont considérés comme ayant un potentiel considérable dans des régions montagneuses reculées, remplaçant progressivement la pomme de terre, aidant à connecter les populations aux marchés rémunérateurs et conduisant à une réduction significative du niveau de pauvreté.
En Asie occidentale, la preuve est donnée par les communautés rurales que la préservation des espèces fruitières vulnérables peut être rémunératrice et qu'en d'autres termes la valeur ajoutée peut ne pas s'opposer à la préservation de la biodiversité. Mais cela ne signifie pas qu'il faut fermer la porte aux productions commerciales d'espèces fruitières comme l'est l'olivier qui se développe fortement dans les zones marginales. Grâce à une approche participative, il est possible de limiter la pression sur les fragiles ressources du milieu.

De telles études de cas en région NENA (Near EastNorth Africa) sont autant d'expériences qui peuvent être partagées avec d'autres régions dans le monde et, en particulier, avec l'Afrique subsaharienne et australe ; dans ces zones, les arbres fruitiers tropicaux et subtropicaux peuvent jouer un rôle important dans la gestion des ressources naturelles et de la biodiversité, de la réduction de la pauvreté, de la sécurité alimentaire et de la santé des populations locales.

Dr. Jacky Ganry

Président de la section Fruits tropicaux et subtropicaux ISHS 